\title{
Eigenfunctions of the offset Fourier, fractional Fourier, and linear canonical transforms
}

\author{
Soo-Chang Pei and Jian-Jiun Ding \\ Department of Electrical Engineering, National Taiwan University, Taipei 10617, Taiwan
}

Received October 11, 2002; accepted October 15, 2002

\begin{abstract}
The offset Fourier transform (offset FT), offset fractional Fourier transform (offset FRFT), and offset linear canonical transform (offset LCT) are the space-shifted and frequency-modulated versions of the original transforms. They are more general and flexible than the original ones. We derive the eigenfunctions and the eigenvalues of the offset FT, FRFT, and LCT. We can use their eigenfunctions to analyze the self-imaging phenomena of the optical system with free spaces and the media with the transfer function $\exp \left[j\left(h_{2} x^{2}+h_{1} x\right.\right.$ $\left.+h_{0}\right)$ (such as lenses and shifted lenses). Their eigenfunctions are also useful for resonance phenomena analysis, fractal theory development, and phase retrieval. (c) 2003 Optical Society of America
\end{abstract}

OCIS codes: $070.2580,070.2590,070.6020$.

\section{INTRODUCTION}

The Fourier transform $(\mathrm{FT})^{1}$ is a well-known transform. The fractional Fourier transform (FRFT) is a generalization of the $\mathrm{FT}^{2-4}$ and the linear canonical transform $(\mathrm{LCT})^{5,6}$ is a further generalization of the FRFT. They are all useful mathematical tools and are widely used for spectrum analysis, signal processing, and optical system analysis.

Offset FTs are similar to the original FTs, except that the kernel $\exp (-j \omega x)$ is replaced by $\exp [-j(\omega-\tau)(x$ $-\eta)$. That is, the kernel is generalized by appending a space-shifted term and a frequency-modulated term. We can also define offset FRFTs and offset LCTs in a similar way. Offset FTs, FRFTs, and LCTs are more flexible than the original ones. They are useful in optics. They are especially useful for analyzing optical systems with prisms or shifted lenses.

The eigenfunctions and the eigenvalues of FTs have been studied, and the eigenfunctions and the eigenvalues of FRFTs and LCTs have also been derived. ${ }^{5,7,8}$ In this paper, we derive the eigenfunctions and the eigenvalues of offset FTs, FRFTs, and LCTs.

Since offset FTs, FRFTs, and LCTs have close relations with the optical system consisting of lenses, free spaces, prisms, and shifted lenses, we can use their eigenfunctions to analyze the self-imaging phenomena of this type of optical system (the eigenfunctions of the original FT, FRFT, and LCT can analyze only the optical system with lenses and free spaces). At the same time, their eigenfunctions are also useful for resonance phenomena analysis, derivation of the eigenvectors of the offset DFT, fractal theory development, mode selection, and phase retrieval.

\section{PRELIMINARIES}

A. Definition of the Original and the Offset Fourier Transform, Fractional Fourier Transform, and Linear Canonical Transform

The well-known Fourier transform (FT) is ${ }^{1}$

$$
G_{F}(\omega)=\mathrm{FT}[g(x)]=\frac{1}{\sqrt{2 \pi}} \int_{-\infty}^{\infty} \exp (-j \omega x) g(x) \mathrm{d} x
$$

It can be generalized into the fractional Fourier transform $(\mathrm{FRFT})^{2-4}$ :

$$
\begin{aligned}
G_{a}(u)= & O_{F}^{\alpha}[g(x)] \\
= & \left(\frac{1-j \cot \alpha}{2 \pi}\right)^{1 / 2} \int_{-\infty}^{\infty} \exp \left(\frac{j}{2} u^{2} \cot \alpha-j u x \csc \alpha\right. \\
& \left.+\frac{j}{2} x^{2} \cot \alpha\right) g(x) \mathrm{d} x
\end{aligned}
$$

The FRFT can be further generalized into the linear canonical transform $(\mathrm{LCT})^{5,6}$ :

$$
\begin{aligned}
G_{(a, b, c, d)}(u)= & O_{F}^{(a, b, c, d)}[g(x)] \\
= & \left(\frac{1}{j 2 \pi b}\right)^{1 / 2} \int_{-\infty}^{\infty} \exp \left(\frac{j}{2} \frac{d}{b} u^{2}-j \frac{u}{b} x\right. \\
& \left.+\frac{j}{2} \frac{a}{b} x^{2}\right) g(x) \mathrm{d} x \quad \text { when } b \neq 0, \\
G_{(a, b, c, d)}(u)= & O_{F}^{(a, b, c, d)}[g(x)] \\
= & \sqrt{d} \exp \left(j c d u^{2} / 2\right) g(d u) \\
& \text { when } b=0,
\end{aligned}
$$

where $a d-b c=1$ must be satisfied. The FT and the FRFT are the special cases of the LCT where $\{a, b, c, d\}$ $=\{0,1,-1,0\}$ and $\{\cos \alpha, \sin \alpha,-\sin \alpha, \cos \alpha\}$ are premultiplied by constant-phase terms:

$$
\begin{aligned}
& \mathrm{FT}[g(x)]=\sqrt{j} O_{F}^{(0,1,-1,0)}[g(x)], \\
& O_{F}^{\alpha}[g(x)]=[\exp (j \alpha)]^{1 / 2} O_{F}^{(\cos \alpha, \sin \alpha,-\sin \alpha, \cos \alpha)}[g(x)] .
\end{aligned}
$$


The Fresnel transform ${ }^{9}$ is also a special case of the LCT (where $\{a, b, c, d\}=\{1, z \lambda / 2 \pi, 0,1\}$ ). The FRFT and the LCT are more general and flexible than the FT, and some problems that cannot be solved by the FT (such as removing chirp noise) will be solved by them. They can be used for filter design, space-variant pattern recognition, phase retrieval, encryption, optical system analysis, ${ }^{10,11}$ spherical mirror system analysis, ${ }^{12}$ gradient index medium analysis, ${ }^{13}$ etc.

The FT, the FRFT, and the LCT can all be generalized a little by including a space-shifted term and a frequencymodulated term. The offset Fourier transform (offset FT) is defined as

$$
\begin{aligned}
G_{F}^{\tau, \eta}(\omega) & =\mathrm{FT}_{\tau, \eta}[g(x)] \\
& =\frac{1}{\sqrt{2 \pi}} \int_{-\infty}^{\infty} \exp [-j(\omega-\tau)(x-\eta)] g(x) \mathrm{d} x .
\end{aligned}
$$

Its inverse operation, i.e., the inverse offset Fourier transform (offset IFT), is defined as

$$
\begin{aligned}
g(x) & =\operatorname{IFT}_{\tau, \eta}\left[G_{F}^{\tau, \eta}(\omega)\right] \\
& =\frac{1}{\sqrt{2 \pi}} \int_{-\infty}^{\infty} \exp [j(x-\eta)(\omega-\tau)] G_{F}^{\tau, \eta}(\omega) \mathrm{d} \omega .
\end{aligned}
$$

Similarly, the offset fractional Fourier transform (offset FRFT) is defined as

$$
\begin{aligned}
G_{\alpha}^{\tau, \eta}(u)= & O_{F}^{\alpha, \tau, \eta}[g(x)] \\
= & \left(\frac{1-j \cot \alpha}{2 \pi}\right)^{1 / 2} \exp (j \eta u) \int_{-\infty}^{\infty} \\
& \times \exp \left[\frac{j}{2}(u-\tau)^{2} \cot \alpha-j(u-\tau) x \csc \alpha\right. \\
& \left.+\frac{j}{2} x^{2} \cot \alpha\right] g(x) \mathrm{d} x
\end{aligned}
$$

and the offset linear canonical transform (offset LCT) is defined as

$$
\begin{aligned}
G_{(a, b, c, d)}^{\tau, \eta}(u)= & O_{F}^{(a, b, c, d, \tau, \eta)}[g(x)] \\
= & \left(\frac{1}{j 2 \pi b}\right)^{1 / 2} \exp (j \eta u) \int_{-\infty}^{\infty} \\
& \times \exp \left[\frac{j}{2} \frac{d}{b}(u-\tau)^{2}-j \frac{u-\tau}{b} x\right. \\
& \left.+\frac{j}{2} \frac{a}{b} x^{2}\right] g(x) \mathrm{d} x \quad \text { when } b \neq 0, \\
G_{(a, b, c, d)}^{\tau, \eta}(u)= & O_{F}^{(a, 0, c, d, \tau, \eta)}[g(x)] \\
= & \sqrt{d} \exp \left[j \eta u+\frac{j}{2} c d(u-\tau)^{2}\right] g(d u \\
& -d \tau), \quad \text { when } b=0,
\end{aligned}
$$

where

$$
a d-b c=1 \text {. }
$$

The offset LCT is also called the special affine Fourier transformation $^{6}$ or the inhomogeneous canonical transform. ${ }^{14,15}$ The offset FT, FRFT, and LCT are similar to the original FT, FRFT, and LCT, except for the presence of two extra parameters $\tau$ and $\eta$, which correspond to space shifting and frequency modulation, respectively. The offset FT, FRFT, and LCT have the following relations with the original transforms:

$$
\begin{aligned}
G_{F}^{\tau, \eta}(\omega) & =\exp [j(\omega-\tau) \eta] G_{F}(\omega-\tau), \\
G_{\alpha}^{\tau, \eta}(u) & =\exp (j \eta u) G_{\alpha}(u-\tau), \\
G_{(a, b, c, d)}^{\tau, \eta}(u) & =\exp (j \eta u) G_{(a, b, c, d)}(u-\tau) .
\end{aligned}
$$

The offset FRFT and LCT are additive. The additivity property of the offset FRFT is

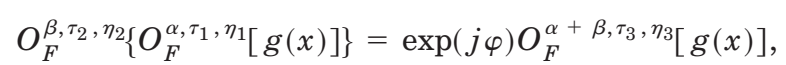

where

$$
\begin{aligned}
\tau_{3}= & \tau_{2}+\tau_{1} \cos \alpha+\eta_{1} \sin \alpha \\
\eta_{3}= & \eta_{2}-\tau_{1} \sin \alpha+\eta_{1} \cos \alpha, \\
\varphi= & \frac{\sin (2 \beta)}{4}\left(\tau_{1}^{2}-\eta_{1}^{2}\right)+\tau_{1} \eta_{1} \sin ^{2} \beta \\
& +\left(\tau_{1} \sin \beta-\eta_{1} \cos \beta\right) \tau_{2} .
\end{aligned}
$$

The additivity property of the offset LCT is

$$
\begin{aligned}
O_{F}^{\left(a_{2}, b_{2}, c_{2}, d_{2}, \tau_{2}, \eta_{2}\right)}\left\{O_{F}^{\left(a_{1}, b_{1}, c_{1}, d_{1}, \tau_{1}, \eta_{1}\right)}[f(x)]\right\} \\
=\exp (j \phi) O_{F}^{(e, f, g, h, r, s)}[f(x)],
\end{aligned}
$$

where

$$
\begin{aligned}
{\left[\begin{array}{ll}
e & f \\
g & h
\end{array}\right]=} & {\left[\begin{array}{ll}
a_{2} & b_{2} \\
c_{2} & d_{2}
\end{array}\right]\left[\begin{array}{ll}
a_{1} & b_{1} \\
c_{1} & d_{1}
\end{array}\right], } \\
\left(\begin{array}{l}
r \\
s
\end{array}\right)= & {\left[\begin{array}{ll}
a_{2} & b_{2} \\
c_{2} & d_{2}
\end{array}\right]\left(\begin{array}{l}
\tau_{1} \\
\eta_{1}
\end{array}\right)+\left(\begin{array}{l}
\tau_{2} \\
\eta_{2}
\end{array}\right), } \\
\phi= & -\frac{a_{2} c_{2}}{2} \tau_{1}^{2}-b_{2} c_{2} \tau_{1} \eta_{1}-\frac{b_{2} d_{2}}{2} \eta_{1}^{2} \\
& -\left(\tau_{1} c_{2}+\eta_{1} d_{2}\right) \tau_{2} .
\end{aligned}
$$

We usually use

$$
\left[\begin{array}{ll}
a & b \\
c & d
\end{array}\right]\left(\begin{array}{l}
t \\
\omega
\end{array}\right)+\left(\begin{array}{l}
\tau \\
\eta
\end{array}\right)
$$

to represent the offset LCT with six parameters $\{a, b, c, d$, $\tau, \eta\},{ }^{6}$ and the combination of the offset LCTs with parameters $\left\{a_{1}, b_{1}, c_{1}, d_{1}, \tau_{1}, \eta_{1}\right\}$ and $\left\{a_{2}, b_{2}, c_{2}, d_{2}, \tau_{2}\right.$, $\left.\eta_{2}\right\}$ can be represented by

$$
\begin{aligned}
{\left[\begin{array}{ll}
a_{2} & b_{2} \\
c_{2} & d_{2}
\end{array}\right]\left\{\left[\begin{array}{ll}
a_{1} & b_{1} \\
c_{1} & d_{1}
\end{array}\right]\left(\begin{array}{c}
t \\
\omega
\end{array}\right)+\left(\begin{array}{l}
\tau_{1} \\
\eta_{1}
\end{array}\right)\right\} } & +\left(\begin{array}{l}
\tau_{2} \\
\eta_{2}
\end{array}\right) \\
& =\left[\begin{array}{ll}
e & f \\
g & h
\end{array}\right]\left(\begin{array}{c}
t \\
\omega
\end{array}\right)+\left(\begin{array}{l}
r \\
s
\end{array}\right),
\end{aligned}
$$


where $e, f, g, h, r$, and $s$ can be calculated from Eqs. (16). From the additivity property, we can conclude that the inverse operation of the offset LCT with parameters $\{a, b, c, d, \tau, \eta\}$ is just the offset LCT with parameters $\{d,-b,-c, a,-d \tau+b \eta, c \tau-a \eta\}$ multiplied by some constant phase:

$$
\begin{aligned}
\left\{O_{F}^{(a, b, c, d, \tau, \eta)}[f(x)]\right\}^{-1} & \\
= & \exp \left(\frac{j}{2} c d \tau^{2}-j a d \tau \eta+\frac{j}{2} a b \eta^{2}\right) \\
& \times O_{F}^{(d,-b,-c, a,-d \tau+b \eta, c \tau-a \eta)}[f(x)] .
\end{aligned}
$$

The offset FT, FRFT, and LCT have two more parameters than the original FT, FRFT, and LCT. They are more flexible. More analysis work in optics can be done by these offset transforms. They can analyze the optical system with prisms or shifted lenses, or the optical components that have the following transfer function:

$$
h(x)=\exp \left[j\left(h_{2} x^{2}+h_{1} x+h_{0}\right)\right] .
$$

They can also analyze the offset spherical mirror pair system as in Fig. 5 below (in Fig. 5, the centers of the two mirrors are not aligned). These tasks cannot be done by the original transforms.

\section{B. Eigenfunctions of the Original Fourier Transform, Fractional Fourier Transform, and Linear Canonical Transform}

The original FT has many eigenfunctions. For example, the products of Hermite functions and $\exp \left(-x^{2} / 2\right)$ are eigenfunctions of the FT, and the corresponding eigenvalues are $(-j)^{m}$ :

$$
\phi_{m}(x)=\exp \left(-x^{2} / 2\right) H_{m}(x),
$$

where $H_{m}(x)$ is the Hermite polynomial of order $m$,

$$
\mathrm{FT}\left(\phi_{m}(x)\right)=(-j)^{m} \phi_{m}(\omega) .
$$

In addition, the FT also has many other eigenfunctions. For example, the following functions are all eigenfunctions of the FT (see Chap. 22 of Ref. 1):

$$
\sum_{n=-\infty}^{\infty} \delta(x-p \sqrt{2 \pi}), \quad\left|\frac{x}{\sqrt{2 \pi}}\right|^{-1 / 2}, \quad \sec h\left(x \sqrt{\frac{\pi}{2}}\right) \text {. }
$$

Although the FT has a variety of eigenfunctions, it has only four possible eigenvalues: $1,-1, j$, and $-j$.

The eigenfunctions and the eigenvalues of the FRFT have also been found. The products of Hermite polynomials and $\exp \left(-x^{2} / 2\right)$, as in Eq. (22), are also eigenfunctions of the FRFT (Ref. 4):

$$
O_{F}^{\alpha}\left[\phi_{m}(x)\right]=\exp (-j m \alpha) \phi_{m}(x),
$$

where $\phi_{m}(x)$ is defined in Eq. (22). In addition, when $\alpha$ $=2 N \pi / M$, the following functions are also eigenfunctions of the $\mathrm{FRFT}^{16}$ :

$$
\begin{aligned}
\Phi(x) & =\sum_{p=0}^{\infty} a_{p} \phi_{p M+q}(x) \\
q & =0,1, \ldots, M-1, a_{p} \text { 's are constants, }
\end{aligned}
$$

and the corresponding eigenvalue is $\exp (-j 2 q N \pi / M)$.

Recently, the eigenfunctions of the LCT were also derived. ${ }^{5,7,8}$ We summarize the eigenfunctions and the eigenvalues of the LCT as follows ${ }^{8}$ :

- Case $A(|a+d|<2)$

$$
\text { Eigenfunctions: } \quad A \exp \left[\frac{-(1+i \rho) x^{2}}{2 \sigma^{2}}\right] H_{m}\left(\frac{x}{\sigma}\right),
$$

where $H_{m}(x)$ is the Hermite polynomial, $A$ is any constant,

$$
\begin{gathered}
\sigma^{2}=\frac{2|b|}{\left[4-(a+b)^{2}\right]^{1 / 2}}, \quad \rho=\frac{\operatorname{sgn}(b)(a-d)}{\left[4-(a+d)^{2}\right]^{1 / 2}} . \\
\text { Eigenvalues: } \quad \lambda=[\exp (-j \alpha)]^{1 / 2} \exp (-i \alpha m),
\end{gathered}
$$

where

$$
\alpha=\cos ^{-1}\left(\frac{a+d}{2}\right)=\sin ^{-1}\left\{\frac{\operatorname{sgn}(b)}{2}\left[4-(a+d)^{2}\right]^{1 / 2}\right\} .
$$

- Case $B(a+d=2, b=0)$

Eigenfunctions:

$$
\begin{aligned}
\sum_{n=0}^{\infty} A_{n} \delta\left(x-\left(4 n \pi|c|^{-1}+h\right)^{1 / 2}\right) & \\
& +\sum_{m=0}^{\infty} B_{m} \delta\left(x+\left(4 m \pi|c|^{-1}+h\right)^{1 / 2}\right),
\end{aligned}
$$

where $0 \leqslant h<4 \pi /|c|, A_{n}$ 's and $B_{m}$ 's are free to choose.

Eigenvalues: $\quad \lambda=\exp (j c h / 2)$.

- Case $C(a+d=-2, b=0)$ : Case $\mathrm{C}$ is the same as case $\mathrm{B}$, except that $A_{n}=B_{n}$ for all $n$ or $A_{n}=-B_{n}$ for all $n$, and the eigenvalues are changed into $\lambda$ $= \pm(-1)^{1 / 2} \exp (j c h / 2)$.

- Case $D(a+d=2, b \neq 0)$

Eigenfunctions:

$$
\exp \left(j \frac{d-a}{4 b} x^{2}\right) \int_{-\infty}^{\infty} \exp \left[j \frac{(x-q)^{2}}{2 \rho}\right] g(q) \mathrm{d} q,
$$

where

$$
\begin{aligned}
g(x)=\sum_{n=0}^{\infty} C_{n} \exp \left[j x\left(4 n \pi|b|^{-1}+h\right)^{1 / 2}\right] \\
+\sum_{m=0}^{\infty} D_{m} \exp \left[-j x\left(4 m \pi|b|^{-1}+h\right)^{1 / 2}\right],
\end{aligned}
$$

$0 \leqslant h<4 \pi /|b|, C_{n}$ 's, $D_{m}$ 's, and $\rho$ can be arbitrary real-valued constants.

Eigenvalues: $\quad \lambda=\exp (-j b h / 2)$.

- Case $E(a+d=-2, b \neq 0)$ : Case $\mathrm{E}$ is the same as case $\mathrm{D}$, except that $C_{n}=D_{n}$ for all $n$ or $C_{n}=-D_{n}$ for all $n$, and the eigenvalues are changed into $\lambda$ $= \pm(-1)^{1 / 2} \exp (-j b h / 2)$.

- Case $F(a+d>2)$

Eigenfunctions:

$$
\exp \left(\frac{j}{2} \rho x^{2}\right) \int_{-\infty}^{\infty} \exp \left[\frac{j}{2 h}(x-q)^{2}\right] g(q) \mathrm{d} q,
$$


where

$$
\begin{aligned}
\sqrt{\sigma g}(\sigma x) & =\lambda g(x), \\
\rho & =\frac{-2 s c}{s(d-a)+\left[(a+d)^{2}-4\right]^{1 / 2}}, \\
h & =\frac{s b}{\left[(a+d)^{2}-4\right]^{1 / 2}}, \\
\sigma & =\left\{a+d \pm\left[(a+d)^{2}-4\right]^{1 / 2}\right\} / 2, \\
s & =\operatorname{sgn}\left[\sigma-\sigma^{-1}\right] . \\
& \text { Eigenvalues: } \quad \lambda=\sqrt{\sigma g}(\sigma x) / g(x) .
\end{aligned}
$$

- Case $G(a+d<-2)$ : Case $\mathrm{G}$ is the same as case $\mathrm{F}$, except that $g(x)= \pm g(-x)$ must be satisfied, $s$ is changed into $\operatorname{sgn}\left(\sigma^{-1}-\sigma\right)$, and the eigenvalues are changed into $\pm(-1)^{1 / 2} \lambda$.

In this paper we derive the eigenfunctions and the eigenvalues of the offset FT, FRFT, and LCT based on the eigenfunctions and the eigenvalues of the original FT, FRFT, and LCT.

\section{EIGENFUNCTIONS OF THE OFFSET FOURIER TRANSFORM, FRACTIONAL FOURIER TRANSFORM, AND LINEAR CANONICAL TRANSFORM}

\section{A. Eigenfunctions of the Offset Fourier Transform}

We first introduce a useful theorem:

Theorem A: Suppose that the linear operator $O_{C}$ can be decomposed as

$$
O_{C}[f(x)]=\exp (j \varphi) O_{B}\left(O_{A}\left\{O_{B}^{-1}[f(x)]\right\}\right),
$$

where $O_{A}$ and $O_{B}$ are linear operators and $\varphi$ is some constant phase. If $e(x)$ is an eigenfunction of $O_{A}$ and the corresponding eigenvalue is $\lambda$, i.e.,

$$
O_{A}[e(x)]=\lambda e(x),
$$

then $O_{B}[e(x)]$ is an eigenfunction of $O_{C}$ and the corresponding eigenvalue is $\lambda \exp (j \varphi)$ :

$$
\begin{aligned}
O_{C}\left\{O_{B}[e(x)]\right\} & =\exp (j \varphi) O_{B}\left[O_{A}\left(O_{B}^{-1}\left\{O_{B}[e(x)]\right\}\right)\right] \\
& =\exp (j \varphi) O_{B}\left\{O_{A}[e(x)]\right\} \\
& =\lambda \exp (j \varphi) O_{B}[e(x)] .
\end{aligned}
$$

Comparing Eq. (6) with Eq. (9), we can conclude that the offset FT is just the offset LCT with parameters $\{a, b, c, d, \tau, \eta\}=\{0,1,-1,0, \tau, \eta\} \quad$ multiplied by $\sqrt{j} \exp (-j \tau \eta)$ :

$$
\mathrm{FT}_{\tau, \eta}[g(x)]=\sqrt{j} \exp (-j \tau \eta) O_{F}^{(0,1,-1,0, \tau, \eta)}[g(x)] .
$$

We then use Theorem A and the additivity property of the offset LCT [see Eqs. (15)-(19)] to derive the eigenfunctions of the offset FT. We choose $O_{C}$ as the offset FT, choose $O_{A}$ as the original $\mathrm{FT}$, and then choose $O_{B}$ as the combination of space shifting and frequency modulation:

$$
\begin{aligned}
O_{B}[g(x)] & =\exp \left(j h_{2} x\right) g\left(x-h_{1}\right), \\
O_{B}^{-1}[g(x)] & =\exp \left(-j h_{1} h_{2}\right) \exp \left(-j h_{2} x\right) g\left(x+h_{1}\right) .
\end{aligned}
$$

Then $O_{A}$ is just the offset LCT with parameters $\{0,1,-1$, $0,0,0\}$ multiplied by $\sqrt{j,} O_{B}$ is the offset LCT with parameters $\left\{1,0,0,1, h_{1}, h_{2}\right\}$, and $O_{B}^{-1}$ is the offset LCT with parameters $\left\{1,0,0,1,-h_{1},-h_{2}\right\} \quad$ multiplied by $\exp \left(-j h_{1} h_{2}\right)$. So Eq. (37) can be rewritten as

$$
\begin{aligned}
\sqrt{j} \exp (-j \tau \eta) O_{F}^{(0,1,-1,0, \tau, \eta)}[f(x)] \\
=\exp (j \varphi) O_{F}^{\left(1,0,0,1, h_{1}, h_{2}\right)}\left(\sqrt{j} O_{F}^{(0,1,-1,0,0,0)}\right. \\
\left.\quad \times\left\{\exp \left(-j h_{1} h_{2}\right) O_{F}^{\left(1,0,0,1,-h_{1},-h_{2}\right)}[f(x)]\right\}\right) .
\end{aligned}
$$

Then, from the additivity property of the offset LCT [see Eqs. (15)-(19)], we can express Eq. (40) as

$$
\begin{aligned}
{\left[\begin{array}{cc}
0 & 1 \\
-1 & 0
\end{array}\right]\left(\begin{array}{l}
t \\
\omega
\end{array}\right)+\left(\begin{array}{l}
\tau \\
\eta
\end{array}\right) } & =\left[\begin{array}{cc}
0 & 1 \\
-1 & 0
\end{array}\right]\left\{\left(\begin{array}{l}
t \\
\omega
\end{array}\right)+\left(\begin{array}{l}
-h_{1} \\
-h_{2}
\end{array}\right)\right\}+\left(\begin{array}{l}
h_{1} \\
h_{2}
\end{array}\right) \\
& =\left[\begin{array}{cc}
0 & 1 \\
-1 & 0
\end{array}\right]\left(\begin{array}{l}
t \\
\omega
\end{array}\right)+\left(\begin{array}{l}
h_{1}-h_{2} \\
h_{1}+h_{2}
\end{array}\right)
\end{aligned}
$$

So we must choose $h_{1}$ and $h_{2}$ as

$$
h_{1}=(\tau+\eta) / 2, \quad h_{2}=(\eta-\tau) / 2 .
$$

Then $O_{B}$ becomes

$$
O_{B}[g(x)]=\exp \left[\frac{j}{2}(\eta-\tau) x\right] g\left(x-\frac{\tau+\eta}{2}\right) .
$$

Then we calculate the constant phase $\varphi$ in Eq. (40). From Eqs. (17) and (40), we obtain

$$
\begin{aligned}
& \sqrt{j} \exp (-j \tau \eta) O_{F}^{(0,1,-1,0, \tau, \eta)}[f(x)] \\
&=\sqrt{j} \exp \left(j \varphi-j h_{1}^{2}+j h_{1} h_{2}-j h_{1} h_{2}\right) \\
& \times O_{F}^{(0,1,-1,0, \tau, \eta)}[f(x)], \\
& \varphi=(\tau-\eta)^{2} / 4 .
\end{aligned}
$$

From Eqs. (43) and (45) and Theorem A, we obtain the following conclusion:

Eigenfunctions and eigenvalues of the offset FT: If $E(x)$ is an eigenfunction of the original FT and $\lambda$ is its corresponding eigenvalue,

$$
\mathrm{FT}[E(x)]=\lambda E(x),
$$

then the offset FT with parameters $\tau$ and $\eta$ has the eigenfunction

$$
E_{\tau, \eta}(t)=\exp \left[\frac{j}{2}(\eta-\tau) x\right] E\left(x-\frac{\tau+\eta}{2}\right),
$$

and its corresponding eigenvalue is $\lambda \exp \left[j(\tau-\eta)^{2} / 4\right]$ :

$$
F_{\tau, \eta}\left[E_{\tau, \eta}(x)\right]=\lambda \exp \left[\frac{j}{4}(\tau-\eta)^{2}\right] E_{\tau, \eta}(x) .
$$

So we can obtain the eigenfunctions and the eigenvalues of the offset FT easily from the eigenfunctions and the eigenvalues of the original FT. In Eq. (47), we can choose $E(x)$ as the Hermite polynomial multiplied by $\exp \left(-x^{2} / 2\right)$ [as in Eq. (22)], or the functions in Eq. (24), or any other eigenfunction of the original FT. From Eq. (26), all the eigenfunctions of the original FT can be expressed as 


$$
\exp \left(-x^{2} / 2\right) \sum_{h=0}^{\infty} a_{h} H_{4 h+q}(x),
$$

$q=0,1,2,3, a_{h}$ 's can be arbitrary real-valued

constants, (49)

where $H_{m}(x)$ is the Hermite polynomial of order $m$, and their corresponding eigenvalues are $(-j)^{q}$. Thus we can conclude that all the eigenfunctions of the offset FT can be expressed as

$$
\begin{aligned}
\Phi_{q, \tau, \eta}^{a_{0}, a_{1}, a_{2}, \cdots}(x)= & \exp \left[j \frac{\eta-\tau}{2} x-\frac{(2 x-\eta-\tau)^{2}}{8}\right] \\
& \times \sum_{h=0}^{\infty} a_{h} H_{4 h+q}\left(x-\frac{\eta+\tau}{2}\right) \\
q= & 0,1,2,3, a_{h} \text { 's are free to choose }
\end{aligned}
$$

and their corresponding eigenvalues are

$$
(-j)^{q} \exp \left[j(\tau-\eta)^{2} / 4\right], \quad q=0,1,2,3 .
$$

Just as the original FT, the offset FT has only four different eigenvalues.

There are some interesting special cases worth noting. From Eq. (47), we find that when $\tau=-\eta$, the amplitude of the eigenfunctions of the offset FT are the same as those of the original FT:

$$
\left|E_{\tau, \eta}(x)\right|=|E(x)| \quad \text { when } \tau=-\eta .
$$

In addition, when

$$
\tau=\eta \text { or, more generally, }(\tau-\eta)^{2}=2 N \pi,
$$

where $N$ is an integer, the eigenvalues of the offset FT are \pm 1 and $\pm j$, which are all the same as those of the original FT.

The eigenfunctions and the eigenvalues of the offset IFT [defined as in Eq. (7)] can also be derived easily. Since the offset IFT is the inverse operation of the offset FT, its eigenfunctions are all the same as those of the offset FT [see Eq. (47) or (50)], and the corresponding eigenvalues are changed from $\lambda$ to $\lambda^{-1}$ (i.e., the corresponding eigenvalues are $j^{q} \exp \left[-(\tau-\eta)^{2} / 4\right]$, where $\left.q=0,1,2,3\right)$.

\section{B. Eigenfunctions of the Offset Linear Canonical} Transform and Fractional Fourier Transform

We also apply Theorem A, described in Subsection 3.A, to derive the eigenfunctions and the eigenvalues of the offset linear canonical transform (offset LCT) with parameters $\{a, b, c, d, \tau, \eta\}$. In Eq. (35), we choose $O_{A}$ as the original LCT with parameters $\{a, b, c, d\}$. We also choose $O_{B}$ and $O_{B}^{-1}$ as the forms (39). Then $O_{A}, O_{B}$, and $O_{B}^{-1}$ are all special cases of the offset LCT, and Eq. (35) can be rewritten as

$$
\begin{aligned}
O_{F}^{(a, b, c, d, \tau, \eta)}[ & f(x)] \\
= & \exp (j \varphi) O_{F}^{\left(1,0,0,1, h_{1}, h_{2}\right)}\left(O_{F}^{(a, b, c, d, 0,0)}\right. \\
& \left.\times\left\{\exp \left(-j h_{1} h_{2}\right) O_{F}^{\left(1,0,0,1,-h_{1},-h_{2}\right)}[f(x)]\right\}\right) .
\end{aligned}
$$

From the additivity property of the offset LCT [see Eqs. (15)-(19)], Eq. (54) can be represented by

$$
\begin{aligned}
{\left[\begin{array}{ll}
a & b \\
c & d
\end{array}\right]\left(\begin{array}{l}
t \\
\omega
\end{array}\right)+\left(\begin{array}{l}
\tau \\
\eta
\end{array}\right)=} & {\left[\begin{array}{ll}
a & b \\
c & d
\end{array}\right]\left\{\left(\begin{array}{l}
t \\
\omega
\end{array}\right)+\left(\begin{array}{l}
-h_{1} \\
-h_{2}
\end{array}\right)\right\}+\left(\begin{array}{l}
h_{1} \\
h_{2}
\end{array}\right) } \\
= & {\left[\begin{array}{ll}
a & b \\
c & d
\end{array}\right]\left(\begin{array}{c}
t \\
\omega
\end{array}\right) } \\
& +\left(\begin{array}{c}
(1-a) h_{1}-b h_{2} \\
-c h_{1}+(1-d) h_{2}
\end{array}\right)
\end{aligned}
$$

Solving the above equation, we obtain

$$
\begin{aligned}
h_{1}= & \frac{(1-d) \tau+b \eta}{2-a-d}, \\
h_{2}= & \frac{c \tau+(1-a) \eta}{2-a-d} \quad(\text { if } a+d \neq 2), \\
O_{B}[g(x)]= & \exp \left[j \frac{c \tau+(1-a) \eta}{2-a-d} x\right] \\
& \times g\left(x-\frac{(1-d) \tau+b \eta}{2-a-d}\right) .
\end{aligned}
$$

Moreover, from Eqs. (17) and (54), we obtain

$$
\begin{aligned}
O_{F}^{(a, b, c, d, \tau, \eta)}[f(x)] \\
=\exp \left[j \varphi+j\left(c h_{1}^{2}+d h_{1} h_{2}\right)-j\left(\frac{a c}{2} h_{1}^{2}+b c h_{1} h_{2}\right.\right. \\
\left.\left.\quad+\frac{b d}{2} h_{2}^{2}-j h_{1} h_{2}\right)\right] O_{F}^{(a, b, c, d, \tau, \eta)}[f(x)] \\
\varphi=\left(\frac{a c}{2}-c\right) h_{1}^{2}+(b c-d+1) h_{1} h_{2}+\frac{b d}{2} h_{2}^{2} \\
=\frac{-c \tau^{2}+2(1-d) \tau \eta+b \eta^{2}}{2(2-a-d)} .
\end{aligned}
$$

From Eqs. (57) and (59) and Theorem A, we obtain the following conclusions:

Eigenfunctions and eigenvalues of the offset LCT: If $E(x)$ is an eigenfunction of the original LCT with parameters $\{a, b, c, d\}$ belonging to $\lambda$, i.e.,

$$
O_{F}^{(a, b, c, d)}[E(x)]=\lambda E(x),
$$

where the form of $E(x)$ is shown in expressions (27)-(34), then the offset LCT with parameters $\{a, b, c, d, \tau, \eta\}$ has the eigenfunction

$$
\begin{aligned}
E_{\tau, \eta}(x)= & \exp \left[j \frac{c \tau+(1-a) \eta}{2-a-d} x\right] \\
& \times E(x \\
& \left.-\frac{(1-d) \tau+b \eta}{2-a-d}\right) \quad(\text { if } a+d \neq 2),
\end{aligned}
$$

and the corresponding eigenvalue is 


$$
\lambda_{\tau, \eta}=\exp \left[j \frac{-c \tau^{2}-2(1-d) \tau \eta+b \eta^{2}}{2(2-a-d)}\right] \lambda .
$$

So the eigenfunctions and the eigenvalues of the offset LCT with parameters $\{a, b, c, d, \tau, \eta\}$ can be obtained easily from those of the original LCT with parameters $\{a, b, c, d\}$. The eigenfunctions of the original LCT are listed in expressions (27)-(34). After a space shift and a frequency modulation, they become the eigenfunctions of the offset LCT.

The above discussion is suitable for the case where $a$ $+d \neq 2$. When $a+d=2$, the above results are incorrect, and we must use other methods to find the eigenfunctions and the eigenvalues.

We first discuss the case where $a+d=2$ and $b=0$. Since $a d-b c=1$ must be satisfied, it follows that $a$ $=d=1$ and the parameters must be of the form $\{a, b, c, d, \tau, \eta\}=\{1,0, c, 1, \tau, \eta\}$. The offset LCT with parameters $\{1,0, c, 1, \tau, \eta\}$ is

$$
O_{F}^{(1,0, c, 1, \tau, \eta)}[g(x)]=\exp \left[j \eta x+j c(x-\tau)^{2} / 2\right] g(x-\tau) .
$$

Thus, when $a+d=2, b=0$, and $\tau \neq 0$, and if $E_{c, \tau, \eta}(x)$ satisfies the quadratic-periodic relation, given by

$$
\lambda E_{c, \tau, \eta}(x)=\exp \left[j \eta x+j c(x-\tau)^{2} / 2\right] E_{c, \tau, \eta}(x-\tau)
$$$$
(\tau \neq 0)
$$

or

$$
\begin{array}{r}
E_{c, \tau, \eta}(x+\tau)=\lambda^{-1} \exp \left[j \eta(x+\tau)+j c x^{2} / 2\right] E_{c, \tau, \eta}(x) \\
(\tau \neq 0),
\end{array}
$$

then $E_{c, \tau, \eta}(x)$ is the eigenfunction of the offset LCT and the corresponding eigenvalue is $\lambda$. In addition, when $a$ $+d=2, b=0$, and $\tau=0$, the eigenfunctions have the form of an impulse train:

When $c \neq 0$ :

$$
\begin{aligned}
\sum_{n=0}^{\infty} A_{n} \delta\left(x+\frac{\eta}{c}-\right. & \left.\left(\frac{4 n \pi}{|c|}+h\right)^{1 / 2}\right) \\
& +\sum_{m=0}^{\infty} B_{m} \delta\left(x+\frac{\eta}{c}+\left(\frac{4 m \pi}{|c|}+h\right)^{1 / 2}\right),
\end{aligned}
$$

$0 \leqslant h<4 \pi /|c|, A_{n}$ 's and $B_{m}$ 's can be arbitrary realvalued constants.

When $c=0$ :

$$
\sum_{n=-\infty}^{\infty} A_{n} \delta\left(x-2 n \pi \eta^{-1}+h\right),
$$

$h$ and $A_{n}$ 's can be arbitrary real-valued constants.

The corresponding eigenvalue is $\exp \left[-j \eta^{2} /(2 c)+j c h / 2\right]$ when $c \neq 0$ and $\exp (-j \eta h)$ when $c=0$.

We then use the above result to derive the eigenfunctions of the offset LCT when $a+d=2$ and $b \neq 0$. In Theorem A, we can choose $O_{A}$ as the offset LCT with parameters $\left\{1,0, c_{1}, 1, \tau_{1}, \eta_{1}\right\}$ and choose $O_{B}$ as the offset LCT with parameters $\left\{a_{2}, b_{2}, c_{2}, d_{2}, \tau_{2}, \eta_{2}\right\}$. Then
$O_{B}^{-1}$ is the original LCT with parameters $\left\{d_{2},-b_{2}\right.$, $\left.-c_{2}, a_{2},-d_{2} \tau_{2}+b_{2} \eta_{2}, c_{2} \tau_{2}-a_{2} \eta_{2}\right\}$, and Eq. (35) can be represented by

$$
\begin{aligned}
& {\left[\begin{array}{ll}
a & b \\
c & d
\end{array}\right]\left(\begin{array}{l}
t \\
\omega
\end{array}\right)+\left(\begin{array}{l}
\tau \\
\eta
\end{array}\right)=} {\left[\begin{array}{ll}
a_{2} & b_{2} \\
c_{2} & d_{2}
\end{array}\right]\left([ \begin{array} { c c } 
{ 1 } & { 0 } \\
{ c _ { 1 } } & { 1 }
\end{array} ] \left\{\left[\begin{array}{cc}
d_{2} & -b_{2} \\
-c_{2} & a_{2}
\end{array}\right]\left(\begin{array}{l}
t \\
\omega
\end{array}\right)\right.\right.} \\
&\left.\left.+\left(\begin{array}{c}
-d_{2} \tau_{2}+b_{2} \eta_{2} \\
c_{2} \tau_{2}-a \eta_{2}
\end{array}\right)\right\}+\left(\begin{array}{l}
\tau_{1} \\
\eta_{1}
\end{array}\right)\right) \\
&+\left(\begin{array}{c}
\tau_{2} \\
\eta_{2}
\end{array}\right), \\
& {\left[\begin{array}{ll}
a & b \\
c & d
\end{array}\right]=} {\left[\begin{array}{cc}
1+c_{1} b_{2} d_{2} & -c_{1} b_{2}{ }^{2} \\
c_{1} d_{2}{ }^{2} & 1-c_{1} b_{2} d_{2}
\end{array}\right] } \\
&\left(\begin{array}{l}
\tau \\
\eta
\end{array}\right)= {\left[\begin{array}{ll}
a_{2} & b_{2} \\
c_{2} & d_{2}
\end{array}\right]\left(\begin{array}{c}
\tau_{1} \\
\eta_{1}
\end{array}\right) } \\
&+\left[\begin{array}{cc}
-a+1 & -b \\
-c & -d+1
\end{array}\right]\left(\begin{array}{l}
\tau_{2} \\
\eta_{2}
\end{array}\right)
\end{aligned}
$$

We solve $c_{1}, b_{2}$, and $d_{2}$ as

$$
\begin{aligned}
& b_{2}= \pm \sqrt{\left|b c_{1}^{-1}\right|}, \\
& d_{2}=\frac{a-d}{2 c_{1} b_{2}},
\end{aligned}
$$

$c_{1}$ can be an arbitrary real-valued constant, except that

$$
\operatorname{sign}\left(c_{1}\right)=-\operatorname{sign}(b) .
$$

In addition, $a_{2}, \tau_{2}$, and $\eta_{2}$ are all free to choose, $c_{2}$ $=\left(a_{2} d_{2}-1\right) / b_{2}=1$, and $\tau_{1}$ and $\eta_{1}$ must be chosen to satisfy Eqs. (69). After some complicated calculation, we can show that, in fact, $a_{2}$ and $\eta_{2}$ have no effect on the eigenfunctions that we obtain. Thus we can fix them as

$$
a_{2}=0, \quad \eta_{2}=0 .
$$

Then the other parameters are

$$
\begin{gathered}
c_{2}=-b_{2}^{-1}, \quad \tau_{2} \text { can be an arbitrary } \\
\text { real-valued constant, } \\
\left.\qquad \begin{array}{c}
\tau_{1} \\
\eta_{1}
\end{array}\right)=\left[\begin{array}{cc}
d_{2} & -b_{2} \\
b_{2}^{-1} & 0
\end{array}\right]\left(\begin{array}{c}
\tau+(a-1) \tau_{2} \\
\eta+c \tau_{2}
\end{array}\right) . \\
\text { Thus we can conclude that the eigenfunctions of the offset } \\
\text { LCT when } a+d=2 \text { and } b \neq 0 \text { are } \\
\qquad E_{\tau, \eta}(x)=\exp \left[j \frac{d_{2}}{2 b_{2}}\left(x-\tau_{2}\right)^{2}\right] \\
\times \int_{-\infty}^{\infty} \exp \left[-\frac{j}{b_{2}}\left(x-\tau_{2}\right) q\right] g(q) \mathrm{d} q,
\end{gathered}
$$$$
\text { real-valued constant, }
$$

where

$$
g\left(x+\tau_{1}\right)=\lambda^{-1} \exp \left[j \eta_{1}\left(x+\tau_{1}\right)+j c_{1} x^{2} / 2\right] g(x),
$$

and the values of $b_{2}, d_{2}, \tau_{2}, c_{1}, \tau_{1}$, and $\eta_{1}$ are chosen according to expressions (69) and (71). The corresponding eigenvalue is

$$
\lambda \exp (j \varphi),
$$

where $\lambda$ is determined by $g(x)$ [see Eq. (73)], and 


$$
\begin{aligned}
\varphi= & \tau_{1} \eta_{1}+b_{2} d_{2} \eta_{1}^{2}+\left(\tau_{1} c_{2}+\eta_{1} d_{2}\right) \tau_{2} \\
& +\frac{\tau_{1}\left(1+\tau_{1} b_{2} d_{2}\right)}{2} \tau_{1}^{2}+\left(1+\tau_{1} b_{2} d_{2}\right) \tau_{1} \eta_{1} \\
& +\left(\tau_{1} c_{2}+\eta_{1} d_{2}\right)\left(b_{2} \eta_{1}+\tau_{2}\right)+\frac{c_{2} d_{2} \tau_{2}^{2}}{2}
\end{aligned}
$$

After the eigenfunctions and the eigenvalues of the offset LCT are derived, we can derive the eigenfunctions and the eigenvalues of the offset FRFT easily. Since the offset FRFT is the special case of the offset LCT with parameters $\{a, b, c, d, \tau, \eta\}=\{\cos \alpha, \sin \alpha,-\sin \alpha, \cos \alpha, \tau, \eta\}$, then, from Eqs. (60)-(62), we obtain the following result:

Eigenfunctions and eigenvalues of the offset FRFT: If $E(x)$ is an eigenfunction of the original FRFT and $\lambda$ is the corresponding eigenvalue,

$$
O_{F}^{\alpha}\langle E(x)\rangle=\lambda E(x),
$$

then the offset FRFT with parameters $\{\alpha, \tau, \eta\}(\alpha \neq 0)$ has the eigenfunction

$$
\begin{aligned}
E_{\tau, \eta}(x)= & \exp \left[j \frac{-\tau \cot (\alpha / 2)+\eta}{2} x\right] \\
& \times E\left[x-\frac{\tau+\eta \cot (\alpha / 2)}{2}\right],
\end{aligned}
$$

and the corresponding eigenvalue is

$$
\lambda_{\tau, \eta}=\exp \left[j \frac{\cot (\alpha / 2)}{4}\left(\tau^{2}+\eta^{2}\right)-j \frac{\tau \eta}{2}\right] \lambda .
$$

The case where $\alpha=0$ is the same as the case of the offset LCT with parameters $\{1,0,0,1, \tau, \eta\}$.

\section{Completion, Orthogonality, and Symmetry Properties}

In any condition, we can find a subset of the eigenfunctions of the offset FT, FRFT, or LCT [denoted by $\left.\left\{\phi_{0}(x), \phi_{1}(x), \phi_{2}(x), \phi_{3}(x), \ldots\right\}\right]$ that satisfies properties (79)-(81):

$$
\int_{-\infty}^{\infty} \phi_{m}(x) \overline{\phi_{n}(x)} \mathrm{d} x=C_{m} \delta_{m, n} .
$$

Any function can be decomposed as a linear combination of the eigenfunctions:

$$
f(x)=\sum_{n=0}^{\infty} a_{n} \phi_{n}(x), \quad \text { and } a_{n}=\frac{\int_{-\infty}^{\infty} f(x) \overline{\phi_{n}(x)} \mathrm{d} x}{\int_{-\infty}^{\infty}\left|\phi_{n}(x)\right|^{2} \mathrm{~d} x}
$$

where $f(x)$ is any function.

$$
\left|\phi_{n}(x)\right|=\left|\phi_{n}\left(-x+x_{0}\right)\right| \quad \text { for all } n,
$$

where $x_{0}$ is some constant.

Thus the offset FT, FRFT, and LCT always have an orthogonal, complete, and symmetric eigenfunction set, whatever the values of their parameters are. That is, for example, for the offset FT, we can choose $\left\{\phi_{0}(x)\right.$, $\left.\phi_{1}(x), \phi_{2}(x), \phi_{3}(x), \ldots\right\}$ as

$$
\begin{aligned}
\phi_{n}(x)= & \exp \left[j \frac{\eta-\tau}{2} x-\left(x-\frac{\tau+\eta}{2}\right)^{2}\right] \\
& \times H_{n}\left(x-\frac{\tau+\eta}{2}\right), \\
n= & 0,1,2,3, \ldots .
\end{aligned}
$$

It indeed forms an orthogonal, complete, and symmetric function set.

\section{ANALYSIS OF SELF-IMAGING PHENOMENA}

The eigenfunctions of the offset FT, FRFT, and LCT are useful for self-imaging phenomena analysis in optics. This is because many optical systems can be modeled by the offset FT, FRFT, and LCT. ${ }^{9-11}$ Before discussing selfimaging phenomena analysis, we first describe how to represent optical systems by the offset FT, FRFT, and LCT:

1. The free space with distance $z$ can be modeled by the offset LCT with parameters

$$
\{a, b, c, d, \tau, \eta\}=\{1, z \lambda / 2 \pi, 0,1,0,0\} .
$$

2. The lens with focal length $f$ can be modeled by the offset LCT with parameters

$$
\{a, b, c, d, \tau, \eta\}=\{1,0,-2 \pi / f \lambda, 1,0,0\} .
$$

3. The prism whose ratio of bottom width to height is $\sigma$ can be modeled by the offset LCT with parameters

$$
\{a, b, c, d, \tau, \eta\}=\{1,0,0,1,0,-2 \pi(n-1) \sigma / \lambda\},
$$

where

$$
\begin{aligned}
& \sigma=\frac{\text { bottom }- \text { (width of the prism) }}{\text { height of the prism }}, \\
& n=\text { refractive index of the prism. }
\end{aligned}
$$

4. The shifted lens in Fig. 1 can be modeled by the offset LCT with parameters

$$
\{a, b, c, d, \tau, \eta\}=\left\{1,0,-2 \pi / f \lambda, 1,0,2 \pi x_{0} / f \lambda\right\} .
$$

5. In addition to the above optical components, there are many other optical components that can be modeled by the offset LCT. For example, suppose that there is a medium (shown as in Fig. 2) with refractive index $n$ and that its width is a second-order function of $x$ ( $x$ axis is perpendicular to the direction of propagation):

$w(x)=h_{2} x^{2}+h_{1} x+h_{0}, \quad x$ is not too large,

then we can represent the medium by the offset LCT with parameters

$$
\begin{aligned}
{\left[\begin{array}{ll}
a & b \\
c & d
\end{array}\right] } & =\left[\begin{array}{cc}
1 & 0 \\
4 \pi(n-1) h_{2} / \lambda & 1
\end{array}\right], \\
\left(\begin{array}{l}
\tau \\
\eta
\end{array}\right) & =\left(\begin{array}{c}
0 \\
2 \pi(n-1) h_{1} / \lambda
\end{array}\right) .
\end{aligned}
$$

If an optical system consists of the optical components described above, then we can use the offset LCT with some parameters set to represent this system. We first 


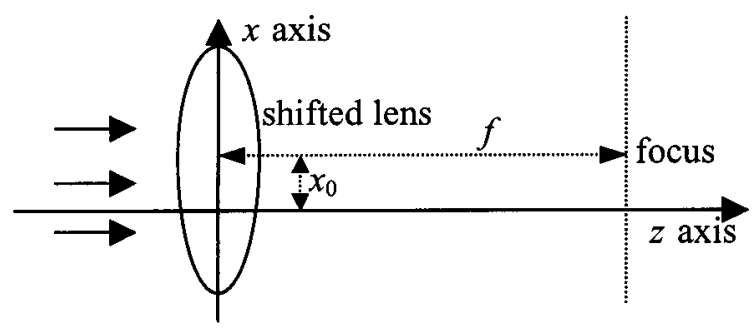

Fig. 1. Lens shifted upward with distance $x_{0}$.

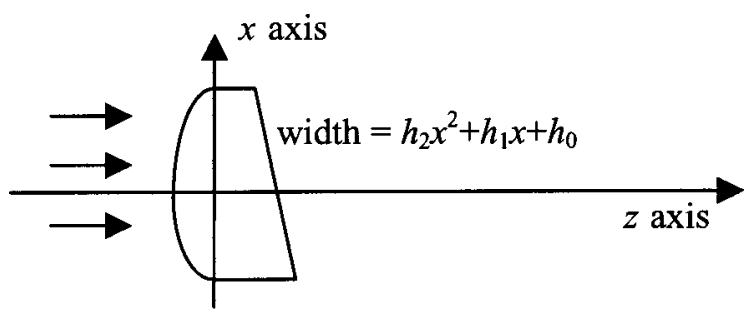

Fig. 2. Medium whose width is $h_{2} x^{2}+h_{1} x+h_{0}$.

represent each of the components (in order from input to output) in the optical system by the offset LCTs with parameter sets $\mathbf{A}_{1}, \mathbf{A}_{2}, \mathbf{A}_{3}, \ldots, \mathbf{A}_{N}$ (each of the $\mathbf{A}_{n}$ 's has the six parameters $\left.\left\{a_{n}, b_{n}, c_{n}, d_{n}, \tau_{n}, \eta_{n}\right\}\right)$. Then the whole optical system can be represented by

$$
\begin{aligned}
D_{o}(x)= & \exp (j \varphi) O_{F}^{(a, b, c, d, \tau, \eta)} \\
& \times\left[D_{i}(x)\right], \quad \varphi \text { is some constant phase, }
\end{aligned}
$$

where $D_{i}(x)$ and $D_{o}(x)$ are the light distributions of the input and the output of the optical system and

$$
\begin{aligned}
O_{F}^{(a, b, c, d, \tau, \eta)} & {[g(x)] } \\
= & O_{F}^{\mathbf{A}_{N}}\left\{O_{F}^{\mathbf{A}_{N-1}}\left[\cdots O_{F}^{\mathbf{A}_{3}}\left(O_{F}^{\mathbf{A}_{2}}\left\{O_{F}^{\mathbf{A}_{1}}[g(x)]\right\}\right) \cdots\right]\right\} .
\end{aligned}
$$

From the additivity property of the offset LCT [see Eqs. (15)-(19)], the values of $\{a, b, c, d, \tau, \eta\}$ can be calculated from a series of matrix operations as in Eq. (19).

If we can use the offset LCT with parameters $\{a, b, c, d, \tau, \eta\}$ to represent an optical system, then the eigenfunctions of the offset LCT with parameters $\left\{a_{1}, b_{1}, c_{1}, d_{1}, \tau_{1}, \eta_{1}\right\}$ can cause the self-imaging phenomena for this optical system if $\left\{a_{1}, b_{1}, c_{1}, d_{1}, \tau_{1}, \eta_{1}\right\}$ satisfy some constraints. From Eq. (9), we can prove that

$$
\begin{aligned}
& \left|\sqrt{\sigma} G_{\left(a_{1}, b_{1}, c_{1}, d_{1}, \tau_{1}, \eta_{1}\right)}\left(\sigma\left(u-\tau+\sigma^{-1} \tau_{1}\right)\right)\right| \\
& \quad=\left|G_{(a, b, c, d, \tau, \eta)}(u)\right| \quad \text { when } \sigma=a_{1} / a=b_{1} / b .
\end{aligned}
$$

Thus we obtain the following conclusions:

1. In optics, only the intensity is observed. So, from Eq. (92), if

$$
a_{1}=a, \quad b_{1}=b, \quad \tau_{1}=\tau,
$$

then, even if $\left\{a_{1}, b_{1}, c_{1}, d_{1}, \tau_{1}, \eta_{1}\right\} \neq\{a, b, c, d$, $\tau, \eta\}$, the eigenfunctions of the offset LCT with parameters $\left\{a_{1}, b_{1}, c_{1}, d_{1}, \tau_{1}, \eta_{1}\right\}$ will cause the self-imaging phenomena for the optical system that can be modeled by the offset LCT with parameters $\{a, b, c, d, \tau, \eta\}$.
2. Furthermore, if the difference of location is ignored, then constraints (93) are relaxed to

$$
a_{1}=a, \quad b_{1}=b
$$

3. If the difference of scaling is ignored, then constraints (93) are relaxed to

$$
a_{1}: b_{1}=a: b, \quad \tau_{1}=a_{1} \tau / a .
$$

4. If both the difference of location and the difference of scaling are ignored, then constraints (93) are relaxed to

$$
a_{1}: b_{1}=a: b
$$

Thus, if an optical system can be modeled by the offset LCT, as in Eq. (90), then there are usually varieties of inputs that can cause self-imaging phenomena.

In Ref. 8, we applied the eigenfunctions of the original LCT to analyze self-imaging phenomena of the optical system consisting of free spaces and lenses. Nevertheless, if an optical system consists of prisms, shifted lenses, and the optical components as in Fig. 2, then its selfimaging phenomena are hard to analyze by the eigenfunctions of the original LCT. This problem can be solved successfully by using the eigenfunctions of the offset LCT.

We give an example in Fig. 3. It has a prism and a shifted lens, so we cannot use the eigenfunctions of the original LCT to analyze self-imaging phenomena. Nevertheless, we can use the eigenfunctions of the offset LCT. From Eqs. (83), (85), and (87), we can model this system by

$$
\begin{gathered}
{\left[\begin{array}{cc}
1 & z_{2} k^{-1} \\
0 & 1
\end{array}\right]\left(\left[\begin{array}{cc}
1 & z_{1} k^{-1} \\
0 & 1
\end{array}\right]\left\{\left[\begin{array}{cc}
1 & 0 \\
-k f^{-1} & 1
\end{array}\right]\left(\begin{array}{c}
t \\
\omega
\end{array}\right)+\left(\begin{array}{c}
0 \\
k x_{0} f^{-1}
\end{array}\right)\right\}\right.} \\
\left.+\left(\begin{array}{cc}
0 \\
-k(n-1) \sigma
\end{array}\right)\right) \\
=\left[\begin{array}{cc}
1-\left(z_{1}+z_{2}\right) f^{-1} & \left(z_{1}+z_{2}\right) k^{-1} \\
-k f^{-1}
\end{array}\right]\left(\begin{array}{c}
t \\
\omega
\end{array}\right) \\
+\left(\begin{array}{c}
\left(z_{1}+z_{2}\right) x_{0} f^{-1}-(n-1) z_{2} \sigma \\
k\left[x_{0} f^{-1}-(n-1) \sigma\right]
\end{array}\right), \\
(k=2 \pi / \lambda) .
\end{gathered}
$$

So the optical system can be modeled by the offset LCT with parameters

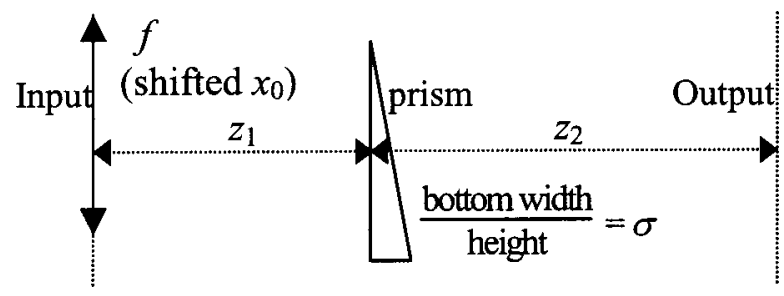

Fig. 3. Optical system with a shifted lens and a prism. 


$$
\begin{aligned}
{\left[\begin{array}{ll}
a & b \\
c & d
\end{array}\right] } & =\left[\begin{array}{cc}
1-\left(z_{1}+z_{2}\right) f^{-1} & \left(z_{1}+z_{2}\right) k^{-1} \\
-k f^{-1} & 1
\end{array}\right], \\
\left(\begin{array}{l}
\tau \\
\eta
\end{array}\right) & =\left(\begin{array}{c}
\left(z_{1}+z_{2}\right) x_{0} f^{-1}-(n-1) z_{2} \sigma \\
k\left[x_{0} f^{-1}-(n-1) \sigma\right]
\end{array}\right) .
\end{aligned}
$$

Then, from Eqs. (93) and (98), we can conclude that if

$$
\begin{aligned}
a_{1} & =1-\left(z_{1}+z_{2}\right) f^{-1}, \quad b_{1}=\left(z_{1}+z_{2}\right) k^{-1}, \\
\tau_{1} & =\left(z_{1}+z_{2}\right) x_{0} f^{-1}-(n-1) z_{2} \sigma,
\end{aligned}
$$

then the eigenfunctions of the offset LCT with parameters $\left\{a_{1}, b_{1}, c_{1}, d_{1}, \tau_{1}, \eta_{1}\right\}$ can cause the self-imaging phenomena for the optical system in Fig. 3. Furthermore, from Eq. (96), if

$$
\frac{a_{1}}{b_{1}}=\frac{2 \pi}{\lambda}\left[\left(z_{1}+z_{2}\right)^{-1}-f_{1}^{-1}\right],
$$

then the eigenfunctions of the offset LCT with parameters $\left\{a_{1}, b_{1}, c_{1}, d_{1}, \tau_{1}, \eta_{1}\right\}$ can cause the self-imaging phenomena if the differences of scaling and location are ignored. In fact,

$$
\begin{aligned}
\left|D_{0}(x)\right| & =\left|\lambda \sqrt{\rho} D_{i}(\sigma(x-s))\right|, \\
\rho & =\frac{a_{1} f}{f-z_{1}-z_{2}}, \\
s & =\frac{\left(z_{1}+z_{2}\right) x_{0}}{f}-(n-1) z_{2} \sigma-\frac{\tau_{1}}{\rho},
\end{aligned}
$$

where $D_{i}(x)$ and $D_{o}(x)$ are the input and the output of the system in Fig. 3 , respectively, and $D_{i}(x)$ satisfies $O_{F}^{\left(a_{1}, b_{1}, c_{1}, d_{1}, \tau_{1}, \eta_{1}\right)}\left[D_{i}(x)\right]=\lambda D_{i}(x)$, where $a_{1}, b_{1}$, and $\tau_{1}$ satisfy Eq. (100).

From the above conclusions, and applying the results in Eqs. (60)-(62), we can find all possible inputs that can cause the self-imaging phenomena for the optical system in Fig. 3. There are varieties of inputs that can cause the self-imaging phenomena for this optical system.

There are two interesting things worth noting:

1. Suppose that $D_{i}(x)$ can cause a self-imaging phenomenon for an optical system. If we shift a lens upward or downward, or place a prism in this optical system, then $D_{i}(x)$ can still cause the self-imaging phenomenon, except that the location of the output image is shifted.

2 . The following input will cause self-imaging phenomena for all the optical systems that have no optical component other than lenses, free spaces, prisms, shifted lenses, or the optical medium in Fig. 2 when the differences of scaling and location are ignored:

$$
D_{i}(x)=A \exp \left(j y_{0} x\right) \exp \left(-\frac{x^{2}}{2}\right) H_{m}\left(x-x_{0}\right),
$$

where $H_{m}(x)$ is the Hermite polynomial of order $m$, and $A, x_{0}$, and $y_{0}$ are free to choose. This is because from Eq. (77), we can conclude that Eq. (103) is the eigenfunction of the offset FRFT with parameters $\alpha, \tau$, and $\eta$, where

$$
\begin{aligned}
& \tau=x_{0}(1-\cos \alpha)-y_{0} \sin \alpha, \\
& \eta=x_{0} \sin \alpha-y_{0}(1-\cos \alpha),
\end{aligned}
$$

where $\alpha$ is any real number. The offset FRFT with these parameters is just the offset LCT with parameters $\left\{\cos \alpha, \sin \alpha,-\sin \alpha, \cos \alpha, x_{0}(1-\cos \alpha)-y_{0} \sin \alpha, x_{0} \sin \alpha\right.$ $\left.-y_{0}(1-\cos \alpha)\right\}$. When the differences of scaling and location are ignored, its eigenfunctions can cause selfimaging phenomena for the optical system that can be modeled by the offset LCT with parameters $\{a, b, c$, $d, \tau, \eta\}$, where

$$
a / b=\cot \alpha .
$$

Since $\alpha$ is any real number, $a / b$ can also be any real number. Thus $D_{i}(x)$ as defined in Eq. (103) can cause selfimaging phenomena for all optical systems that can be modeled by the offset LCT.

\section{ANALYSIS OF RESONANCE PHENOMENA AND OTHER APPLICATIONS}

\section{A. Analysis of Resonance Phenomena}

Analyzing the resonance phenomena of spherical mirror pair systems (as in Figs. 4 and 5) is another application of the eigenfunctions of the offset FT, FRFT, and LCT.

The eigenfunctions of the original FT can cause resonance phenomena for a cofocal spherical mirror pair system (i.e., the system in Fig. 4 when $R_{A}=R_{B}=D$ ). ${ }^{17}$ In our new manuscript in preparation, ${ }^{18}$ we show that the eigenfunctions of the original LCT can cause resonance phenomena for a spherical mirror pair system even when $R_{A} \neq R_{B} \neq D$. The only requirement is that the centers of the two spherical mirrors be aligned, as in Fig. 4 .

In fact, even when the centers of the two mirrors are not aligned together (as in Fig. 5), we can still analyze the resonance phenomena of the spherical mirror pair system. This is because this system can be modeled by the offset LCT. Suppose that, in Fig. 5, the center of mirror A is at $(x, y)=(0,0)$, the center of mirror $\mathrm{B}$ is at $(x, y)$ $=\left(x_{0}, y_{0}\right)\left(\left|x_{0}\right|\right.$ and $\left|y_{0}\right|$ are not too large), and the reflective coefficients of mirrors $\mathrm{A}$ and $\mathrm{B}$ are $r_{A}$ and $r_{B}$, respectively:

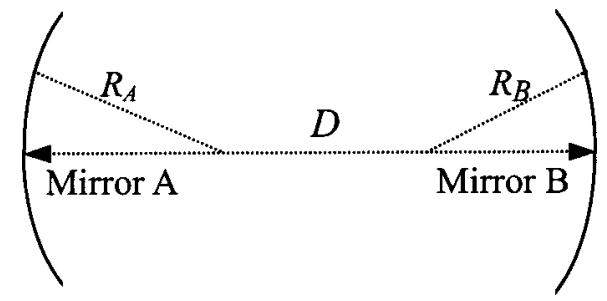

Fig. 4. Spherical mirror pair system (the centers of the two mirrors are aligned).

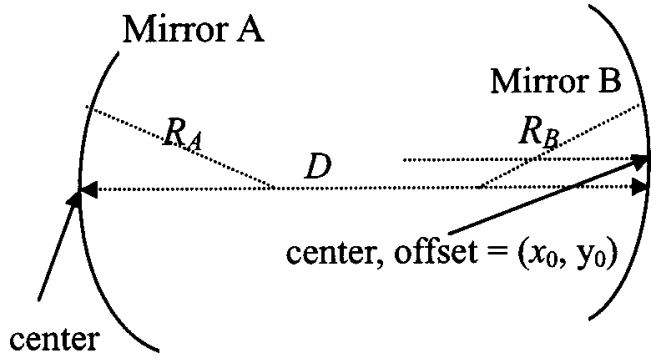

Fig. 5. Spherical mirror pair system (the centers of the two mirrors are not aligned). 


$$
F_{A R}(x, y)=r_{A} F_{A I}(x, y), \quad F_{B R}(x, y)=r_{B} F_{B I}(x, y),
$$

where $F_{A I}(x, y)$ and $F_{B I}(x, y)$ denote the incident light of mirrors $\mathrm{A}$ and $\mathrm{B}$ and $F_{A R}(x, y)$ and $F_{B R}(x, y)$ denote the refractive light of mirrors $\mathrm{A}$ and $\mathrm{B}$.

If the light $F_{i}(x, y)$ is emitted from mirror $\mathrm{A}$, it will propagate rightward to mirror $\mathrm{B}$, be reflected by mirror $\mathrm{B}$, and then propagate back to mirror $\mathrm{A}$. Then the reflective light will be received by mirror $\mathrm{A}$. If we denote the light received by mirror $\mathrm{A}$ as $F_{1}(x, y)$, then

$$
\begin{aligned}
F_{1}(x, y)= & \exp [j(2 k D+\phi)] r_{B} O_{F_{y}}^{\left(a, b, c, d, \tau_{y}, \eta_{y}\right)} \\
& \times\left\{O_{F_{x}}^{\left(a, b, c, d, \tau_{x}, \eta_{x}\right)}\left[F_{i}\left(x^{\prime}, y^{\prime}\right)\right]\right\},
\end{aligned}
$$

where $O_{F_{x}}$ and $O_{F_{y}}$ stand for the one-dimensional offset LCT along the $x$ axis and the $y$ axis, $k=2 \pi / \lambda$, and then propagate backward. The propagation-reflection operation will repeat again and again. If the original input light $F_{i}(x, y)$ has the distribution (110) and we use $F_{n}(x, y)$ to denote the light received by mirror A for the $n$th time, then

$$
F_{n}(x, y)=\exp [j(2 k n D+n \phi)] \zeta_{1} \zeta_{2} r_{A}^{n-1} r_{B}^{n} F_{i}(x, y) .
$$

It is always a constant multiple of $F_{i}(x, y)$. If the coherent constraint is satisfied, i.e.,

$$
\arg \left\{\exp [j(2 k n D+n \phi)] \zeta_{1} \zeta_{2} r_{A} r_{B}\right\}=0,
$$

then the resonance phenomena occur. Thus, if the original input light has the same distribution as that of the eigenfunctions of the offset LCT, as in Eqs. (110) and (111), and its eigenvalues satisfy the coherent constraint (114), then the input light will cause resonance phenomena. Since, usually, $\left|\zeta_{A} \zeta_{B} r_{A} r_{B}\right|<1$, we can prove that the total light received by mirror $\mathrm{A}$ is

$$
\begin{aligned}
{\left[\begin{array}{ll}
a & b \\
c & d
\end{array}\right] } & =\left[\begin{array}{cc}
2\left(1-\frac{D}{R_{A}}\right)\left(1-\frac{D}{R_{B}}\right)-1 & \frac{2 D}{k}\left(\frac{D}{R_{B}}-1\right) \\
2 k\left(\frac{1}{R_{A}}+\frac{1}{R_{B}}-\frac{2 D}{R_{A} R_{B}}-\frac{D}{R_{A}^{2}}+\frac{D^{2}}{R_{A}^{2} R_{B}}\right) & 2\left(1-\frac{D}{R_{A}}\right)\left(1-\frac{D}{R_{B}}\right)-1
\end{array}\right] \\
\left(\begin{array}{l}
\tau_{x} \\
\eta_{x}
\end{array}\right) & =\left(\begin{array}{c}
2 x_{0} D R_{B}^{-1} \\
2 k x_{0} R_{B}^{-1}\left(1-R_{A}^{-1} D\right)
\end{array}\right), \quad\left(\begin{array}{c}
\tau_{y} \\
\eta_{y}
\end{array}\right)=\left(\begin{array}{c}
2 y_{0} D R_{B}^{-1} \\
2 k y_{0} R_{B}^{-1}\left(1-R_{A}^{-1} D\right)
\end{array}\right),
\end{aligned}
$$

$$
\phi=\frac{2 k D\left(x_{0}^{2}+y_{0}^{2}\right)}{R_{B}^{2}}\left(\frac{D}{R_{A}}-1\right) .
$$

That is, the relations between the emitted light and the received reflective light of the spherical mirror pair system in Fig. 5 can be expressed by the offset LCT. If the mirrors are aligned $\left(x_{0}=y_{0}=0\right)$, then, in Eqs. (108), $\tau_{x}=\eta_{x}=\tau_{y}=\eta_{y}=0$, and we can use just the original LCT to model the system. Nevertheless, when $x_{0} \neq 0$ or $y_{0} \neq 0$, we must use the offset LCT to model the system in Fig. 5. is

Thus, in Fig. 5, if the input light emitted from mirror A

$$
F_{i}(x, y)=f_{1}(x) f_{2}(y)
$$

where $f_{1}(x)$ and $f_{2}(y)$ are all the eigenfunctions of the offset LCT, i.e.,

$$
\begin{aligned}
& O_{F_{x}}^{\left(a, b, c, d, \tau_{x}, \eta_{x}\right)}\left[f_{1}\left(x^{\prime}\right)\right]=\zeta_{1} f_{1}(x), \\
& O_{F_{y}}^{\left(a, b, c, d, \tau_{y}, \eta_{y}\right)}\left[f_{2}\left(y^{\prime}\right)\right]=\zeta_{2} f_{2}(y),
\end{aligned}
$$

where $a, b, c, d, \tau_{x}, \eta_{x}, \tau_{y}$, and $\eta_{y}$ are defined as in Eqs. (108), then, in Eq. (107), the light $F_{1}(x, y)$ received by mirror A is just a constant multiple of $F_{i}(x, y)$ :

$$
F_{1}(x, y)=\exp [j(2 k D+\phi)] \zeta_{1} \zeta_{2} r_{B} F_{i}(x, y) .
$$

Furthermore, the received light $F_{1}(x, y)$ will be reflected by mirror $\mathrm{A}$, propagate forward to mirror $\mathrm{B}$ again, and

$$
\begin{aligned}
F_{S}(x, y) & =\sum_{n=1}^{\infty} F_{n}(x, y) \\
& =\frac{\exp [j(2 k D+\phi)] \zeta_{A} \zeta_{B} r_{B}}{1-\exp [j(2 k D+\phi)] \zeta_{A} \zeta_{B} r_{A} r_{B}} F_{i}(x, y) .
\end{aligned}
$$

If $\left|\zeta_{A} \zeta_{B} r_{A} r_{B}\right| \approx 1$ and Eq. (114) is satisfied, then $\left|F_{S}(x, y)\right|$ can be many times $\left|F_{i}(x, y)\right|$.

\section{B. Other Potential Applications}

1. Deriving the Eigenvectors of the Offset Discrete Fourier Transform, Discrete Cosine Transform, and Discrete Sine Transform

The eigenvectors of the discrete Fourier transform (DFT) can be derived from the eigenfunctions of the original FT. $^{19}$ In fact, we can also use the eigenfunctions of the offset FT found in this paper to derive the eigenvectors of the offset DFT, which is defined as

$$
X_{a, b}[m]=\sqrt{N^{-1}} \sum_{n=0}^{N-1} \exp \left[-j \frac{2 \pi}{N}(m-a)(n-b)\right] x[n] .
$$

Moreover, we can further derive the eigenvectors of the discrete cosine transform and the discrete sine transform.

\section{Fractal Analysis}

When $|a+d|>2$, the eigenfunctions of offset LCTs are scaling-invariant functions (i.e., fractals) with some extra 
operations. Thus, developing the fractal theory and analyzing the performance of fractals in optics are also potential applications of the eigenfunctions of offset LCTs.

\section{Mode Selection and Its Related Applications}

A mode selection algorithm for the original FRFT has been developed. ${ }^{16}$ Since the eigenfunctions of the original FRFT are complete and orthogonal, every function $f(x)$ can be expressed as

$$
f(x)=\sum_{n=0}^{\infty} a_{n} \phi_{n}(x),
$$

where $\phi_{n}(x)$ is the eigenfunction of the original FRFT. The mode selection algorithm is the one that uses the FRFTs with different parameters (can be implemented by optical systems) to expand the input function $f(x)$ as in Eq. (117) by optics. ${ }^{16}$ It is useful for retrieval of the phase information in optics, ${ }^{20}$ analysis of the light spectrum, ${ }^{21}$ and encryption. ${ }^{22}$

Since the offset FT, FRFT, and LCT also have a complete and orthogonal eigenfunction set (see Subsection 3.C), we can also use an algorithm similar to the mode selection algorithm of the original FRFT (Ref. 16) to do the work of mode selection in optics. Thus we can also use the eigenfunctions of the offset FT, FRFT, and LCT for the applications of phase retrieval in optics, light spectrum analysis, and encryption.

\section{CONCLUSIONS}

In this paper, we have derived the eigenfunctions and the eigenvalues of the offset Fourier transform (FT), offset fractional Fourier transform (FRFT), and offset linear canonical transform (LCT). The properties and the applications of these eigenfunctions are also discussed. With the aid of the eigenfunctions of the offset FT, FRFT, and LCT, we can study self-imaging phenomena of the optical system consisting of lenses, free spaces, lenses, and shifted lenses, and the media with the transfer functions $\exp \left[j\left(h_{2} x^{2}+h_{1} x+h_{0}\right)\right]$. In addition, we can also use the eigenfunctions of the offset FT, FRFT, and LCT for resonance phenomena analysis, mode selection, phase retrieval, and other potential applications.

\section{ACKNOWLEDGMENTS}

This research was supported by the National Science Council, Taiwan, under contract NSC91-2219-E-002-044, and by the Ministry of Education, Taiwan, under contract 89-E-FA06-2-4.

Corresponding author Soo-Chang Pei can be reached by e-mail, pei@cc.ee.ntu.edu.tw; or fax, 886-2-23671909.

\section{REFERENCES}

1. R. N. Bracewell, The Fourier Integral and Its Applications (McGraw-Hill, Boston, Mass., 2000).

2. L. B. Almeida, "The fractional Fourier transform and timefrequency representations," IEEE Trans. Signal Process. 42, 3084-3091 (1994)

3. H. M. Ozaktas, M. A. Kutay, and Z. Zalevsky, The Fractional Fourier Transform with Applications in Optics and Signal Processing (Wiley, New York, 2000).

4. V. Namias, "The fractional order Fourier transform and its application to quantum mechanics," J. Inst. Math. Appl. 25, 241-265 (1980).

5. K. B. Wolf, "Canonical transforms," in Integral Transforms in Science and Engineering, K. B. Wolf, ed. (Plenum, New York, 1979), Chap. 9, pp. 381-416.

6. S. Abe and J. T. Sheridan, "Optical operations on wave functions as the Abelian subgroups of the special affine Fourier transformation," Opt. Lett. 19, 1801-1803 (1994).

7. D. F. V. James and G. S. Agarwal, "The generalized Fresnel transform and its applications to optics," Opt. Commun. 126, 207-212 (1996).

8. S. C. Pei and J. J. Ding, "Eigenfunctions of linear canonical transform," IEEE Trans. Signal Process. 50, 11-26 (2002).

9. J. W. Goodman, Introduction to Fourier Optics (McGrawHill, New York, 1996).

10. M. J. Bastiaans, "Propagation laws for the second-order moments of the Wigner distribution function in first-order optical systems," Optik (Stuttgart) 82, 173-181 (1989).

11. H. M. Ozaktas and D. Mendlovic, "Fractional Fourier optics," J. Opt. Soc. Am. A 12, 743-751 (1995).

12. P. Pellat-Finet and G. Bonnet, "Fractional order Fourier transform and Fourier optics," Opt. Commun. 111, 141-154 (1994)

13. D. Mendlovic, H. M. Ozaktas, and A. W. Lohmann, "Gradedindex fibers, Wigner distribution and the fractional Fourier transform," Appl. Opt. 33, 6188-6193 (1994).

14. S. Abe and J. T. Sheridan, "Generalization of the fractional Fourier transformation to an arbitrary linear lossless transformation: an operator approach," J. Phys. A 27, 4179-4187 (1994).

15. S. Abe and J. T. Sheridan, "Corrigenda to 'Generalization of the fractional Fourier transformation to an arbitrary linear lossless transformation: an operator approach', J. Phys. A 27, 7937-7938 (1994)

16. T. Alieva and A. M. Barbe, "Self-fractional Fourier functions and selection of modes," J. Phys. A 30, 211-215 (1997).

17. S. G. Lipson and H. Lipson, Optical Physics (Cambridge U. Press, Cambridge, UK, 1981).

18. S. C. Pei and J. J. Ding, Department of Electrical Engineering, National Taiwan University, No. 1, Sec. 4, Roosevelt Road, Taipei 10617, Taiwan, are preparing a manuscript to be called "Properties and applications of the eigenfunctions of the linear canonical transform."

19. M. L. Mehta, "Eigenvalues and eigenvectors of the finite Fourier transform," J. Math. Phys. 28, 781-785 (1987).

20. T. Alieva and M. J. Bastiaans, "Finite-mode analysis by means of intensity information on fractional optical systems," J. Opt. Soc. Am. A 19, 481-484 (2002).

21. T. Alieva and M. J. Bastiaans, "Mode analysis in optics through fractional transforms," Opt. Lett. 24, 1206-1208 (1999).

22. T. Alieva and M. J. Bastiaans, "Powers of transfer matrices determined by means of eigenfunctions," J. Opt. Soc. Am. A 16, 2413-2418 (1999). 